\title{
The Application of Resettable Device to Semi-Active Tuned Mass Damper Building Systems for Multi-level Seismic Hazard Mitigation
}

\author{
Min-Ho Chey \\ School of Architecture and Art, Yanbian University of Science and Technology, Yanji, PR China
}

http://dx.doi.org/10.5659/AIKAR.2012.14.3.99

\begin{abstract}
An innovative multi-story Semi-Active Tuned Mass Damper (SATMD) building system is proposed to control seismic response of existing structures. The application of adding new stories as large tuned mass and semi-active (SA) resettable actuators as central features of the control scheme is derived. For the effective control of the structures, the optimal tuning parameters are considered for the large mass ratio, for which a previously proposed equation is used and the practical optimal stiffness is allocated to the actuator stiffness and rubber bearing stiffness. A two-degree-of freedom (2-DOF) model is adopted to verify the principal efficiency of the suggested structural control concept. The simulations for this study utilizes the three ground motions, from SAC project, having probability of exceedance of $50 \%$ in 50 years, $10 \%$ in 50 years, and 2\% in 50 years for the Los Angeles region. 12-story moment resisting frames, which are modified as ' $12+2$ ' and ' $12+4$ ' story structures, are investigated to assess the viability and effectiveness of the system that aims to reduce the response of the buildings to earthquakes. The control ability of the SATMD scheme is compared to that of an uncontrolled and an ideal Passive Tuned Mass Damper (PTMD) building system. From the performance results of suggested ' $12+2$ ' and ' $12+4$ ' story retrofitting case studies, SATMD systems shows significant promise for application of structural control where extra stories might be added.
\end{abstract}

\section{Keywords: Resettable Device, Tuned Mass Damper, Semi-Active Control, Seismic Isolation, Multi-level Seismic Hazard}

\section{INTRODUCTION}

Tuned Mass Damper (TMD) systems are one of the widely adopted classic structural control strategy, especially for the high-rise building structures. However, some disadvantages of a TMD system have been found: (i) the limitation of a TMD mass ratio to the effectiveness of the TMD (usually the size); (ii) the narrow band control related sensitivity; and (iii) the fluctuation ability in tuning frequencies between the TMD and a controlled structure. To overcome the limitations regarding the TMD mass ratio, it has been suggested to use a portion of the building itself as a tuned mass damper. Villaverde (2002) and Tian et al. (2008) utilized the sliding roof systems to isolate a 13 -story and an 8-story framed structure from earthquake excitations respectively. The papers showed that the proposed implementations of using modified isolation system has the good potential to become a practical and effective way to reduce seismic damage in low- and medium-rise buildings with alleviating the necessity for additional mass. Meanwhile, Pan et al. (1995), Pan and Cui (1995), and Charng (1998) sought to evaluate the effect of using segmental structures where isolation devices are placed at various heights in the structure, as well as at the base. Each segment may comprise a few stories and is

Corresponding Author : Min-Ho Chey, Associate Professor

School of Architecture and Art, Yanbian University of Science and

Technology, Yanji, PR China

3458 Chaoyang St., Yanji City, Jilin Province, China, 133000

Tel: +864332695119 e-mail : hnhdad@yust.edu

This is an Open Access article distributed under the terms of the Creative Commons Attribution Non-Commercial License (http://creativecommons. org/licenses/by-nc/3.0/) which permits unrestricted non-commercial use, distribution, and reproduction in any medium, provided the original work is properly cited. interconnected by additional vibration isolation systems. The good of these systems is to reduce the seismic demand rather than increasing the earthquake resistance capacity of the structure. Proper application of these technologies leads to better performing structures that will remain essentially during various levels of earthquakes.

As an alternative way of avoiding the limitations of the TMD regarding the control band and turning ability, semi-active (SA) structural control method has been emerged for mitigating structural damage over active and passive solutions. SA systems are strictly dissipative and do not add energy to the system, guaranteeing stability. As one of the damper-based SA control strategies, Bobrow and Jabbari (1997), and Jabbari and Bobrow (2002) have previously focused on the basic analytical techniques needed to characterize structural systems that use a resettable SA device for vibration suppression. Furthermore, Hunt (2002), Barroso et al. (2003) and Chase et al. (2004) have investigated the ability of semi-active resettable devices to mitigate structural response in the presence of hysteretic, geometric and yielding nonlinearities under various intensity level seismic hazard suite.

In this paper, a multi-story semi-active tuned mass damper (SATMD) building system is suggested as an advanced structural isolation method. A resettable device is used as a SA operation and some stories are newly added on a given uncontrolled structure. For this study, the dynamic characteristics and seismic linear elastic responses of the 2-DOF model are investigated to examine the effectiveness of the suggested control system. For the multi-degreeof freedom (MDOF) application, ' $12+2$ ' and ' $12+4$ ' story SATMD building models are adopted and these response results are compared with those from the corresponding uncontrolled (No TMD) and passive tuned mass damper (PTMD) building systems. For the realistic assessment, three ground motion accelerations having different intensity level seismic hazard are used. 


\section{PTMD \& SATMD BUILDING SYSTEMS}

\subsection{Design Concept and Simplified Modeling}

The concept of a newly suggested SATMD building system can be viewed as an extension of the conventional TMD system. Various possible design methods may be used to locate the device between two separated segments of the structure to prevent the occurrence of rocking modes of the structure and to transmit the gravity loads between two segments (Charng, 1998). Figure 1 shows a fundamental design concept of the interface level and two different hystereses for viscous damping and resettable device. The upper portion of added stories is passively or semi-actively interconnected to the existing stories by using a viscous damper or a resettable device respectively with rubber bearings as depicted in Figure 1.

The fundamental mechanism of suppressing structural vibration induced by an earthquake is to transfer the vibration energy of the existing structure to the isolated and tunable upper stories. Thus, the seismic force of the entire superstructure can be reduced through the passive or semi-actively operated isolation interface. The overall effectiveness depends on the amount of energy transferred, the size of the added mass (stories) and the ability of the isolating elements used (viscous damper or resettable device).

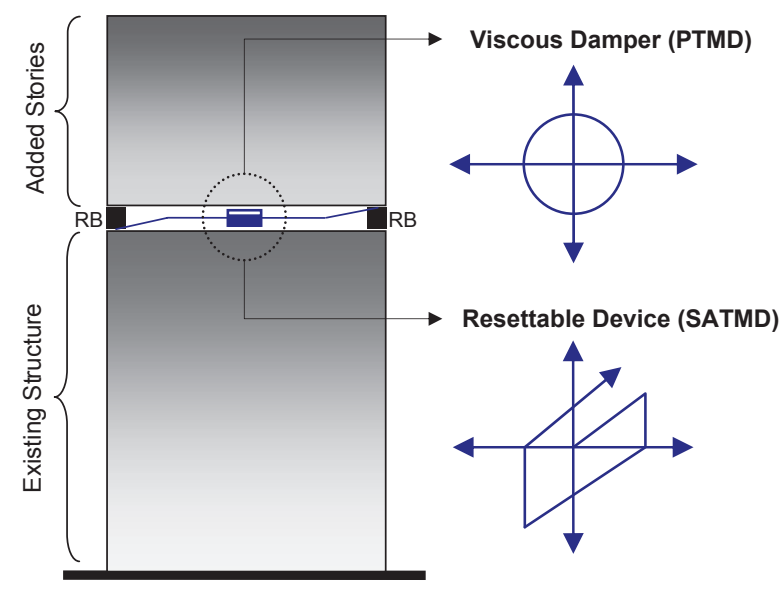

Figure 1. Schematic of design concept

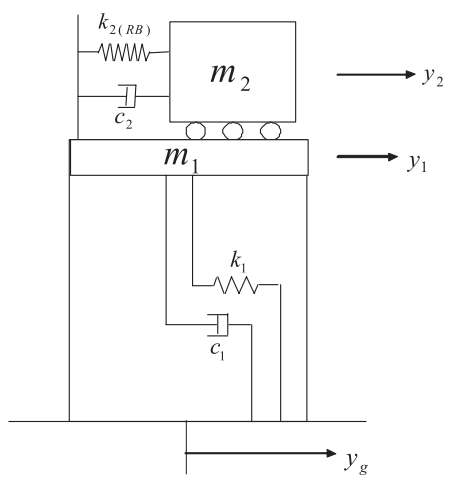

(a) PTMD

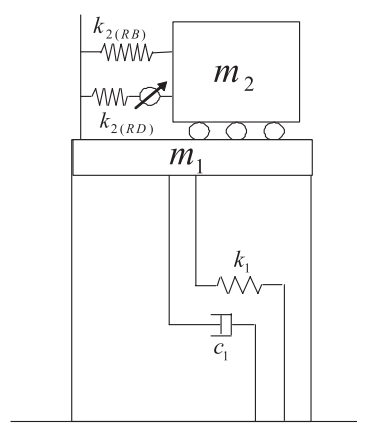

(b) SATMD
Figure 2. Main Systems with PTMD and SATMD

For the simplified modeling of the TMD building system, a two-degree-of freedom (2-DOF) model is used (Figure 2). From the simplified model, the equations of motion of the PTMD and SATMD system subjected to the earthquake load can be derived as

$$
\begin{aligned}
& {\left[\begin{array}{cc}
m_{1} & 0 \\
0 & m_{2}
\end{array}\right]\left\{\begin{array}{l}
\ddot{y}_{1} \\
\ddot{y}_{2}
\end{array}\right\}+\left[\begin{array}{cc}
c_{1}+c_{2} & -c_{2} \\
-c_{2} & c_{2}
\end{array}\right]\left\{\begin{array}{l}
\dot{y}_{1} \\
\dot{y}_{2}
\end{array}\right\}+} \\
& {\left[\begin{array}{cc}
k_{1}+k_{2(R B)} & -k_{2(R B)} \\
-k_{2(R B)} & k_{2(R B)}
\end{array}\right]\left\{\begin{array}{l}
y_{1} \\
y_{2}
\end{array}\right\}=\left\{\begin{array}{l}
-m_{1} \\
-m_{2}
\end{array}\right\} \ddot{y}_{g}} \\
& {\left[\begin{array}{cc}
m_{1} & 0 \\
0 & m_{2}
\end{array}\right]\left\{\begin{array}{l}
\ddot{y}_{1} \\
\ddot{y}_{2}
\end{array}\right\}+\left[\begin{array}{ll}
c_{1} & 0 \\
0 & 0
\end{array}\right]\left\{\begin{array}{l}
\dot{y}_{1} \\
\dot{y}_{2}
\end{array}\right\}+} \\
& {\left[\begin{array}{cc}
k_{1}+k_{2(R B)}+k_{2(R D)} & -k_{2(R B)}-k_{2(R D)} \\
-k_{2(R B)}-k_{2(R D)} & k_{2(R B)}+k_{2(R D)}
\end{array}\right]\left\{\begin{array}{l}
y_{1} \\
y_{2}
\end{array}\right\}=} \\
& \left\{\begin{array}{l}
-m_{1} \\
-m_{2}
\end{array}\right\} \ddot{y}_{g}
\end{aligned}
$$

respectively, in which,

- $m_{1}$ : mass of main system,

- $m_{2}$ : mass of TMD,

- $k_{1}$ : stiffness of main system,

- $k_{2(R B)}$ : stiffness of rubber bearing (PTMD \& SATMD),

- $k_{2(R D)}$ : stiffness of resettable device (SATMD),

- $c_{1}$ : damping coefficient of main system,

- $c_{2}$ : damping coefficient of TMD,

- $y_{1}$ : displacement of main system, relative to the ground,

- $y_{2}$ : motion of TMD, relative to the ground,

- $\ddot{y}_{g}$ : ground acceleration (earthquake loading)

\subsection{Parametric Optimization for Large TMD}

To achieve optimal performance of TMD systems in buildings, the optimal parameters such as the frequency tuning ratio and damping ratio of the TMD need to be determined. Warburton (1982) presented some formulated optimal TMD parameters for various responses. Especially, Sadek et al. (1997) carried the applicability of larger mass TMD for seismic applications and presented the optimal TMD parameters by simple equations using curve-fitting methods.

The equation for the optimal frequency tuning ratio, $f_{2 o p t}$ and the optimal damping ratio, $\xi_{2 o p t}$ of the TMD building system are Equations (3) and (4), in which $\mu=$ mass ratio of the TMD to the main system and $\xi_{1}=$ damping ratio of the main system. For practical application, it is necessary to obtain applicable parameters for the TMD system such as the optimal TMD stiffness, kzopt and optimal damping coefficient, c2opt and these parameters can be derived from $f_{2 o p t}$ and $\xi_{2 o p t}$ as shown in Equations (5) and (6).

$$
f_{2 o p t}=\frac{1}{1+\mu}\left(1-\xi_{1} \sqrt{\frac{\mu}{1+\mu}}\right)
$$




$$
\begin{gathered}
\xi_{2 o p t}=\frac{\xi_{1}}{1+\mu}+\sqrt{\frac{\mu}{1+\mu}} \\
k_{2 o p t}=\left(\frac{m_{2} \omega_{1}^{2}}{1+\mu}\right)^{2}\left(1-\xi_{1} \sqrt{\frac{\mu}{1+\mu}}\right)^{2} \\
c_{2 o p t}=\frac{2 m_{2} \omega_{1}}{1+\mu}\left(1-\xi_{1} \sqrt{\frac{\mu}{1+\mu}}\right)\left(\frac{\xi_{1}}{1+\mu}+\sqrt{\frac{\mu}{1+\mu}}\right)
\end{gathered}
$$

Chey et al. (2010) adopted the equations (3) to (6) to find the optimal parameters for high values of mass ratio. The parametric results indicated that a TMD with both larger stiffness and larger damping is needed, as the larger the mass ratio becomes and there was no more increase in the TMD stiffness when the mass ratio is over 1.0

For the optimal TMD parameters for MDOF structures, Sadek et al. found that the tuning ratio for a MDOF system is nearly equal to the tuning ratio for a 2-DOF system for a mass ratio of $\mu \Phi$, where $\Phi$ is the amplitude of the first mode of vibration for a unit modal participation factor computed at the location of the TMD. The equation for the tuning ratio is obtained from Equation (3) by replacing $\mu$ by $\mu \Phi$ and the equation for the damping ratio is obtained by multiplying Equation (4) by $\Phi$ as shown in equations (7) and (8) respectively. For MDOF structures, the practical parameters of the optimal TMD stiffness and the optimal damping coefficient can therefore be derived as equations (9) and (10).

$$
\begin{gathered}
f_{M 2 \text { opt }}=\frac{1}{1+\mu \Phi}\left(1-\xi_{1} \sqrt{\frac{\mu \Phi}{1+\mu \Phi}}\right) \\
\xi_{M 2 o p t}=\Phi\left(\frac{\xi_{1}}{1+\mu}+\sqrt{\frac{\mu}{1+\mu}}\right) \\
k_{M 2 o p t}=\frac{m_{2} \omega_{1}^{2}}{(1+\mu \Phi)^{2}}\left(1-\xi_{1} \sqrt{\frac{\mu \Phi}{1+\mu \Phi}}\right)^{2} \\
c_{M 2 o p t}=\frac{2 m_{2} \omega_{1}}{1+\mu \Phi}\left(1-\xi_{1} \sqrt{\frac{\mu \Phi}{1+\mu \Phi}}\right)\left(\frac{\xi_{1}}{1+\mu}+\sqrt{\frac{\mu}{1+\mu}}\right)
\end{gathered}
$$

\section{SEMI-ACTIVE RESETTABLE DEVICE}

\subsection{Mechanics of Resettable Device}

Resettable devices act as pneumatic spring element, resisting displacement in either direction, and they possess the ability to release the stored spring energy at any time, creating the semiactive aspect of these devices (Bobrow et al., 2000). Schematically, energy is stored in the device by compressing the air, as the piston is displaced from its center position. When the piston reaches its maximum displaced position in a given cycle, the stored energy is also at a maximum and the device changes direction of motion. Thus, the reset criteria are determined to be the point of zero velocity at displacement peaks. At this point, the stored energy is released by discharging the working air to the non-working side of the device, thus resetting the equilibrium position of the device.

\subsection{Resettable Device with Independent Chambers}

Unlike previously mentioned conventional resettable devices (Figure 3(a)), the resettable device suggested in this research eliminates the need to rapidly dissipate energy from one side of the device to the other by using a two-chambered design that utilizes each piston side independently. This approach treats each side of the piston as an independent chamber with its own valve and control rather than coupling them with a connecting valve (Figure 3(b)). This double-value approach allows a wider variety of control laws to be imposed, as each valve can be operated independently. Thus, independent control of the pressure on each side of the piston is enabled, allowing a greater diversity of device behaviors (Chase et al., 2006; Rodgers et al., 2007).

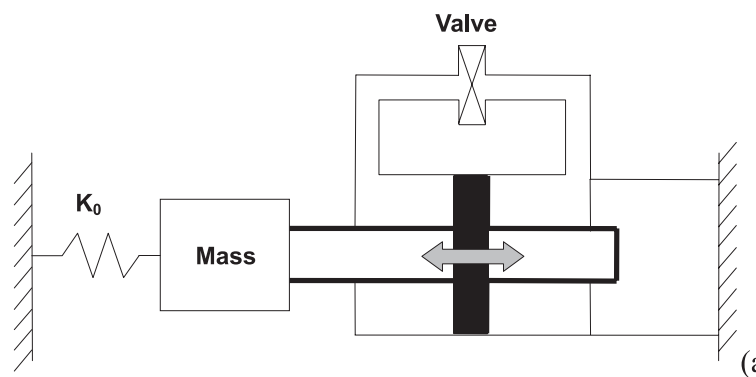

(a)

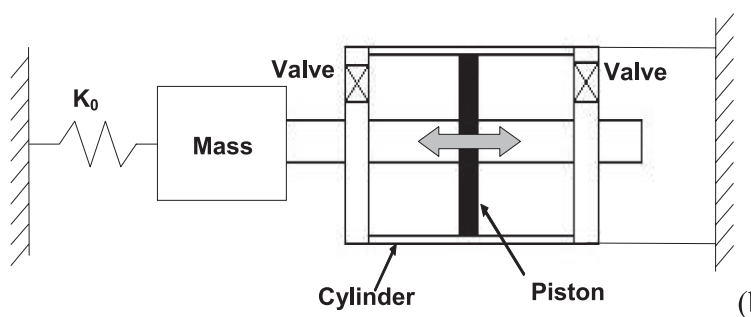

(b)

Figure 3. Schematic of resettable devices attached to a single degreeof-freedom system: (a) a conventional resettable device; and (b) an independent chamber resettable device (Rodgers et al., 2007).

Given that air is an ideal gas it obeys the law:

$$
p V^{r}=c
$$

where $\mathrm{r}$ is the ratio of specific heats, $\mathrm{c}$ is a constant, and $\mathrm{p}$ and $\mathrm{V}$ are, respectively, the pressure and volume in one chamber of the device. If the piston is centered in the device and the initial pressure po in both chambers with initial volumes $V_{0}$, the resisting force is defined as a function of displacement, $\mathrm{x}$ :

$$
\begin{aligned}
F(x) & =\left(p_{2}-p_{1}\right) A c \\
& =\left[\left(V_{0}+A x\right)^{-r}-\left(V_{0}-A x\right)^{-r}\right] A c
\end{aligned}
$$


where A is the piston area. Equation (12) can be linearized and an approximate force defined:

$$
F(x)=-\frac{2 A^{2} r p_{0}}{V_{0}} x
$$

Hence, the effective stiffness of the resettable device, $k 2(R D)$, is readily defined:

$$
k_{2(R D)}=-\frac{2 A^{2} r p_{0}}{V_{0}}
$$

A one-fifth scale pneumatic-based prototype device with independent chambers has been constructed at the department of mechanical engineering, University of Canterbury, to enable experimental testing and device characterization (Mulligan et al., 2005). Mulligan et al. modified the ideal model of Equations (11) to (14) using experimental results to obtain a more realistic, non-linear device model. The dimensions of the one-fifth scale prototype device are described in Figure 4.

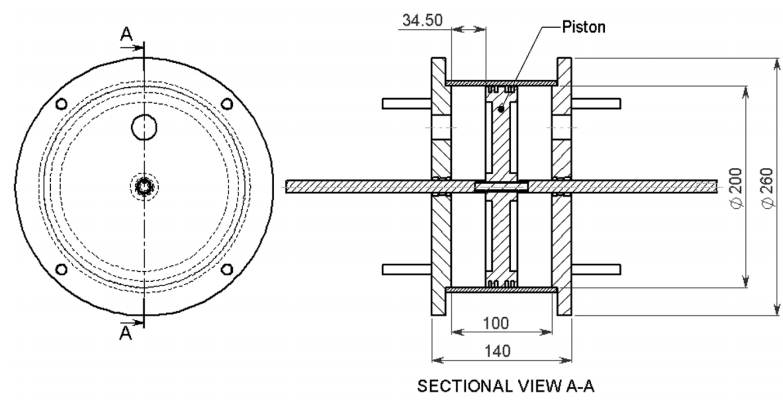

Figure 4. Dimensioned drawing of the prototype resettable device with independent chambers (Mulligan et al., 2005).

\subsection{Modeling in Ruaumoko}

To represent the effects of the resettable device properly, a 'SemiActive Resettable Actuator Member' has been developed for the inelastic dynamic analysis program, Ruaumoko (Carr, 2007). The hysteretic behavior shows that the force is proportional to the displacement until a saturation force is attained, Fy+ or Fy-, which might be near the yield forces for the member (Figure 5(a)). At these values the resisting force is capped by gradually opening the valve, and the system appears to show a perfectly plastic response. On any reversal of displacement the force is automatically reset to zero, the origin is moved to the existing displacement, and the system will then behave as an elastic member until either saturation is reached or the displacement again changes sign. This form is denoted a '1-2-3-4 device' as it provides damping in all quadrants (Chase et al., 2006; Chey et al., 2010).

The hysteretic operation of the resettable device is flexible, offering a wide variety of customized responses. Figure 5(b) shows the force-displacement response if only motion away from zero is resisted. Thus, the law is termed the ' $1-3$ ' law as resistive forces are provided in only the first and third quadrants. Typical peak forces produced by the device under the ' $1-3$ ' law are less than the peak forces for the '1-2-3-4' law, with the same piston displacement. If the control law is changed such that only motion towards the zero position (form peak values) is resisted, the force-deflection curves that result are shown in Figure 5(c). In this case, device provides damping forces only in quadrants 2 and 4; '2-4' device. Peak forces produced by the device under this control law are again less than the peak forces of the device under the '1-2-3-4' control law, for the same total piston displacement. In addition, the peak forces can be slightly lower again than those for the '1-3' response due to the active chamber volume being relatively large (Rodgers et al., 2007).

For the case of the SATMD system adopted in this study, '1-2-3-4' control law is used to represent the standard or initial operation of resettable device. Figure 6 shows the step-by-step one cycle device operating process of the resettable device under '1-2-3-4' control law. The first column shows the piston displacement with respect to time. The second column shows a diagram of the device indicating the piston motion direction and the valve states. The third column shows the ideal force-displacement response.

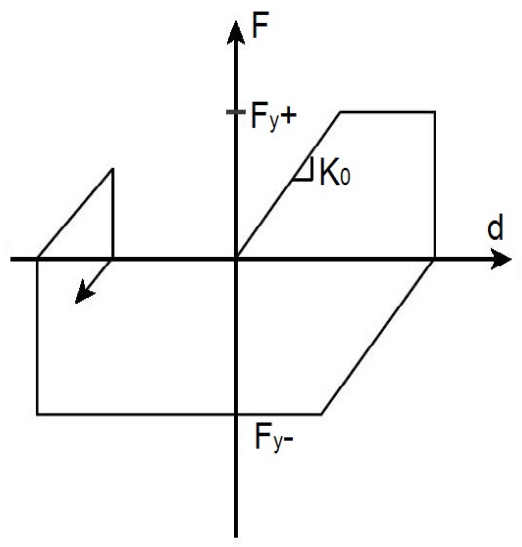

(a) '1-2-3-4' control law

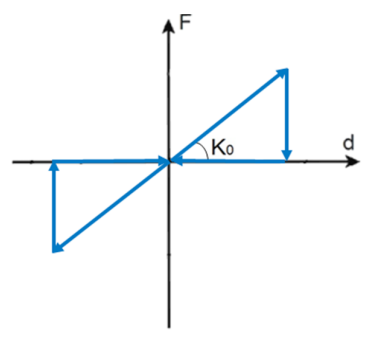

(b) '1-3' control law

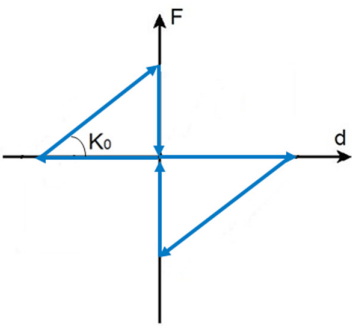

(c) '2-4' control law
Figure 5. Resettable actuator hystereses (Carr, 2007) 
(a)
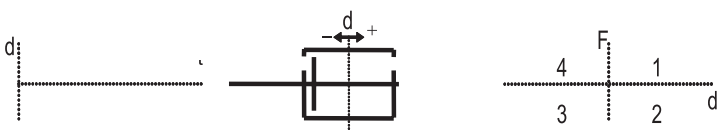

(b)
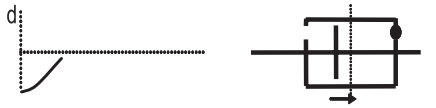

(c)

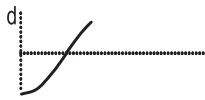

(d)

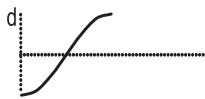

(e)

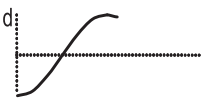

(f)
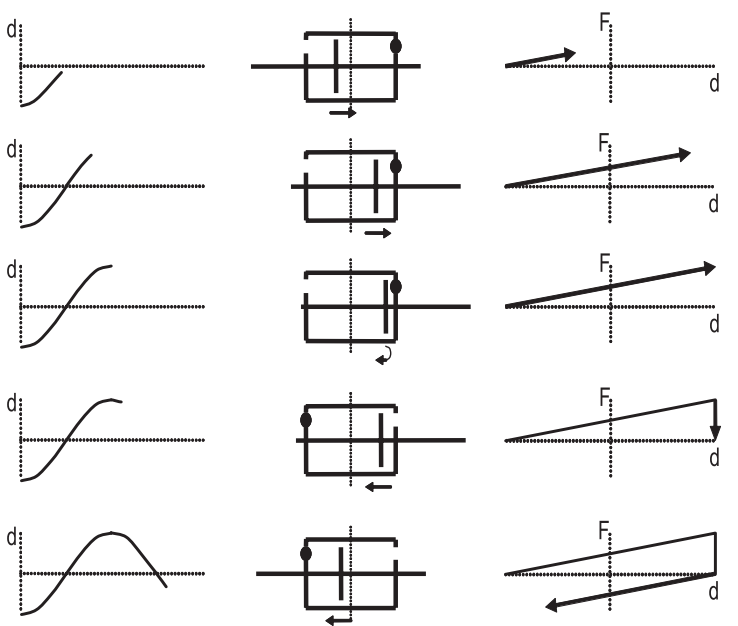

(g)
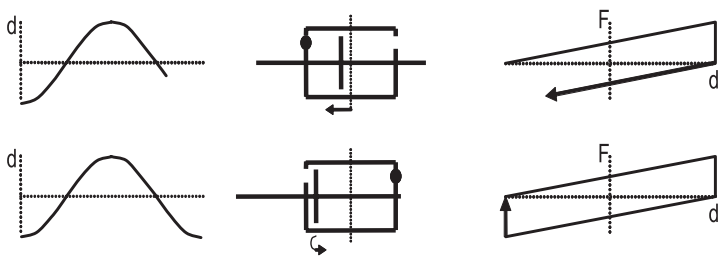

Figure 6. One cycle of the device operation under '1-2-3-4' law

\section{2-DOF MODEL IMPLEMENTATIONS AND GROUND MOTIONS}

To demonstrate the proposed structural control methodology, a 1-DOF linear undamped model (Table 1) is investigated. Note that, it's impossible to find the exact design and optimization criteria for SATMD due to non-linearity of device. As a computationally efficient approach, therefore, we use optimal PTMD stiffness of kzopt and vary its contribution to SATMD systems. Thus, $20 \%, 40 \%, 60 \%$, $80 \%$ and $100 \%$ of PTMD stiffness are allocated to the stiffness of the resettable device to find a (near) optimal value as for an optimallybased parametric design approach. Finally, this overall design approach is computationally simpler and convex compared to nonlinear optimization of each specific controlled structure (Chey et al., 2010).

Performance of system with No TMD, optimal PTMD, and offoptimal PTMD are compared with the five SATMD cases (all 2-DOF models), and the unsaturable force of $40 \mathrm{kN} / \mathrm{m}$ is used as a SA resettable device force. The adopted optimal TMD parameters from Equations (3) to (6) are listed in Table 2. To demonstrate the validity of the optimal parameters, off-optimal PTMD which have the parameters of $\mathrm{f}_{2}=0.625$ and $\xi_{2}=0.612$ were used and these values are the optimal figures for the case of $\mu=0.6$ (larger mass ratio than the optimal PTMD). To illustrate the performance of each control case using the calculated optimal parameters, peak relative displacements and total accelerations of the main system are computed. All response values are shown as percentage ratios from the uncontrolled (No TMD) response as shown in Figures 9 and 10
Table 1. Dynamic properties of undamped main system (1-DOF)

\begin{tabular}{c|c|c}
\hline Item & Main System & Unit \\
\hline Weight & 268 & $\mathrm{kN}$ \\
\hline Effective Mass & 27.3 & $\mathrm{kN}-\mathrm{s}^{2} / \mathrm{m}$ \\
\hline Frequency & 0.5 & $\mathrm{~Hz}$ \\
\hline
\end{tabular}

Table 2. Parameters for TMD building system (2-DOF)

\begin{tabular}{c|c|c|c|c|c}
\hline Model & $\mu$ & $\mathrm{f}_{2 \mathrm{opt}}$ & $\xi_{2 \mathrm{opt}}$ & $\begin{array}{c}\mathrm{k}_{2 \mathrm{opt}} \\
(\mathrm{kN} / \mathrm{m})\end{array}$ & $\begin{array}{c}\mathrm{c}_{2 \text { opt }} \\
(\mathrm{kN}-\mathrm{s} / \mathrm{m})\end{array}$ \\
\hline PTMD & 0.5 & 0.666 & 0.578 & 59.7 & 33.0 \\
\hline SATMD & 0.5 & 0.666 & - & 59.7 & - \\
\hline
\end{tabular}

To reflect the use of multiple time history records over a range of seismic levels, three earthquake ground records representing ground motions having low, medium and high probability of exceedance in 50 years for the Los Angeles area were used (Sommerville et al., 1997) as shown in Table 3. To properly reflect the seismic hazard at the Los Angeles site, each earthquake was scaled. Thus, their response spectra, for a given probability of exceedance, were comparable with the spectrum from the United States Geological Survey (USGS) probabilistic seismic hazard maps for the Los Angeles area, in the period range of 0.3 to 4.0 seconds for stiff local soil conditions.

Figures 7 and 8 show the spectral acceleration and displacement plots for the three different levels of earthquakes used in this study. These spectral acceleration diagrams allow the relative intensity of earthquakes to be assessed, and are developed by determining the response of a 1-DOF system over a spectrum of different periods. Intensity comparison can then be made using the fundamental frequency of the structure of interest.

Table 3. Three earthquakes scaled within various intensity level seismic hazard suites (Sommerville et al., 1997)

\begin{tabular}{c|c|c|c|c|c}
\hline $\begin{array}{c}\text { Probability of } \\
\text { Exceedance }\end{array}$ & Record & Mag. & $\begin{array}{c}\text { Dist. } \\
(\mathrm{km})\end{array}$ & $\begin{array}{c}\text { Scale } \\
\text { Factor }\end{array}$ & $\begin{array}{c}\text { PGA } \\
\left(\mathrm{cm} / \mathrm{sec}^{2}\right)\end{array}$ \\
\hline $\begin{array}{c}50 \% \text { in } 50 \text { yrs. } \\
\text { (Low suite) }\end{array}$ & $\begin{array}{c}\text { Kern } \\
\text { County } \\
(1952)\end{array}$ & 7.7 & 107 & 2.92 & 141.49 \\
\hline $\begin{array}{c}10 \% \text { in } 50 \text { yrs. } \\
(\text { Medium suite })\end{array}$ & $\begin{array}{c}\text { Imperial } \\
\text { Valley } \\
(1940)\end{array}$ & 6.9 & 10 & 2.01 & 452.03 \\
\hline $\begin{array}{c}2 \% \text { in } 50 \text { yrs. } \\
\text { (High suite) }\end{array}$ & $\begin{array}{c}\text { Kobe } \\
(1995)\end{array}$ & 6.9 & 3.4 & 1.15 & 1258.00 \\
\hline
\end{tabular}




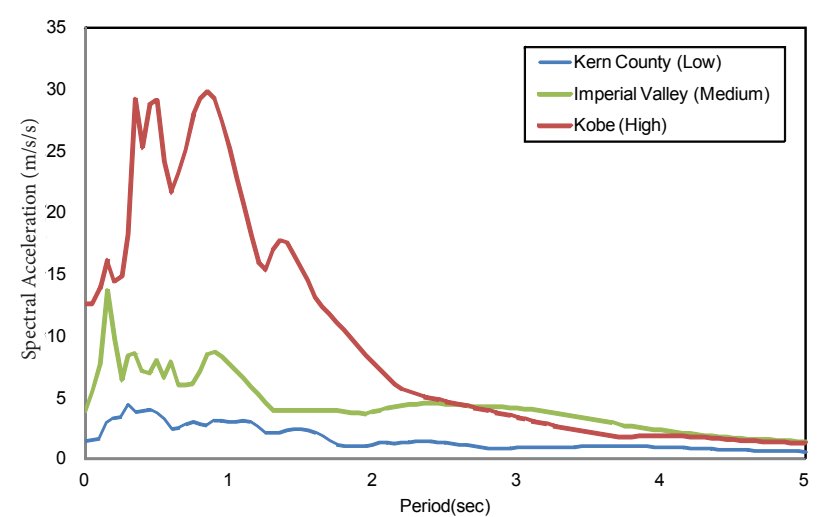

Figure 7. Spectral displacement plots for three earthquakes used ( $5 \%$ critical damping)

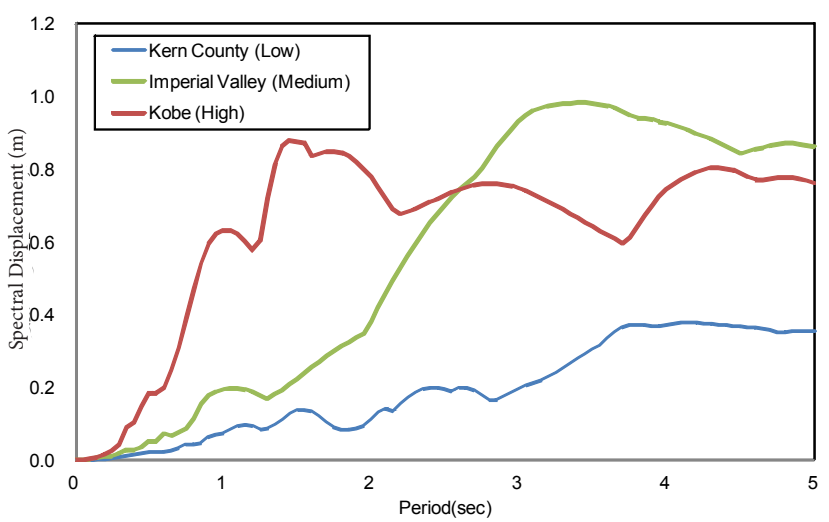

Figure 8. Spectral acceleration plots for three earthquakes used (5\% critical damping)

Overall, from the results in Figures 9 and 10, it is observed that most control cases perform much better than the uncontrolled (No TMD) system. The SATMD building system showed better response reductions in terms of displacement. The PTMD building system presented better reductions in acceleration response, except for the Imperial Valley earthquake (medium suite). As expected, the off-optimal PTMD case produced larger response results on both displacement and acceleration than the optimal PTMD under almost cases and this reflects the mistuned response of the offoptimal PTMD and the validity of the optimal parameters.

The lowest values of displacement and acceleration depend on the earthquakes used, as shown in Figures 9 and 10. For example, the most effective control schemes are SA80TMD (48.1\%) under Kern County (low suite), SA40TMD (62.1\%) under Imperial Valley (medium suite) and SA20TMD (63.7\%) under Kobe (high suite) for displacement response reductions. The effectiveness of TMD building system evidently depends on the frequency content, bandwidth and intensity of the applied motions, as well as the ability to ensure optimal tuning.

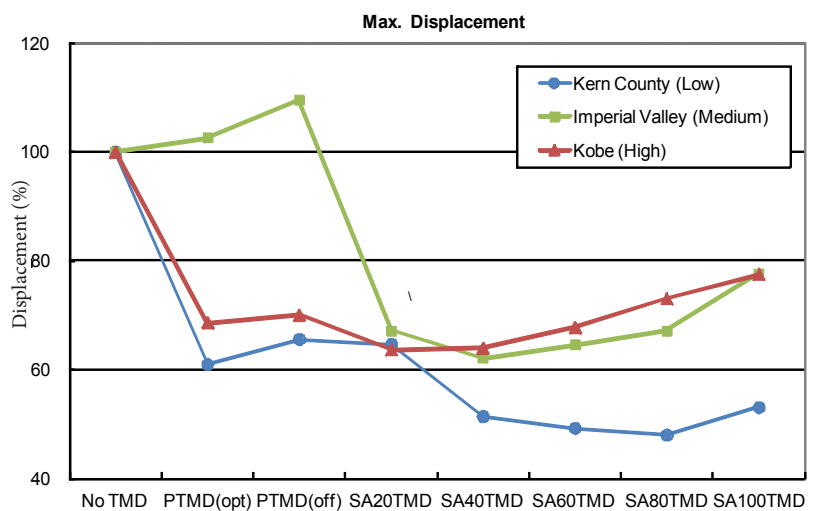

Figure 9. Displacement control effects of PTMD and SATMD

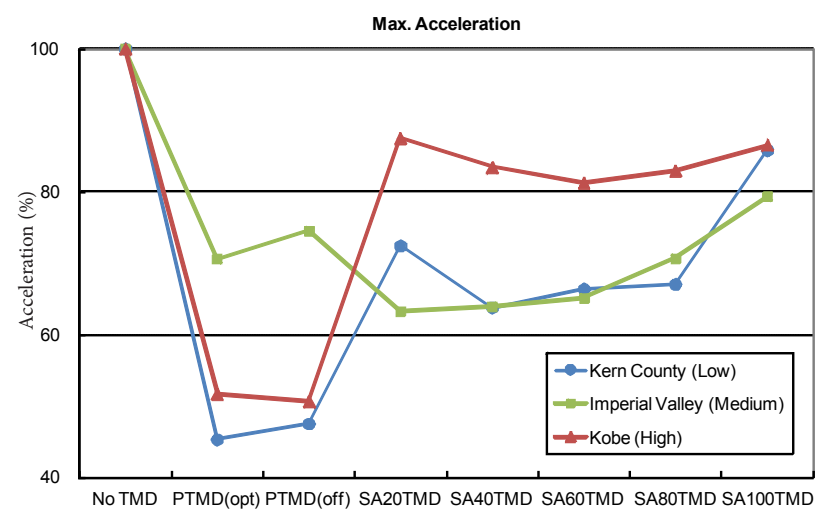

Figure 10. Acceleration control effects of PTMD and SATMD

\section{5. ' $12+2$ ' AND ' $12+4$ ' STORY CASE STUDIES}

\subsection{Multi-Story Structures and modeling}

A 12-story, two-bay reinforced concrete framed structure is used to demonstrate the potential and beneficial effects of TMD building systems as shown in Figure 11. This model was designed originally by Jury (1978) according to the New Zealand Loadings Code (NZS4203, 1976) based on the concept of capacity design. Its strengths were then revised following the changes to NZS4203 made in 1992 (NZS4203, 1992). It was assumed that the frame would be required to resist the component of earthquake motion in the plane of the frame only. No torsional effects for the building as a whole were taken into account. All frames share in carrying gravity and seismic-induced loads, then moment redistribution was carried out using a method developed by Paulay (1976).

The distance between frames is a consistent $9.2 \mathrm{~m}$ for the entire building structure. A width of the floor slab equal to 12 times its thickness was considered to contribute to the elastic stiffness of the beams. The slab thicknesses were $120 \mathrm{~mm}$ for the framed structure. 
The building dimensions adopted in this study are shown in Table 4 and the dynamic properties of the frame are listed in Table 5. The columns above the first level were specified to remain elastic in accordance with the strong column-weak beam concept.

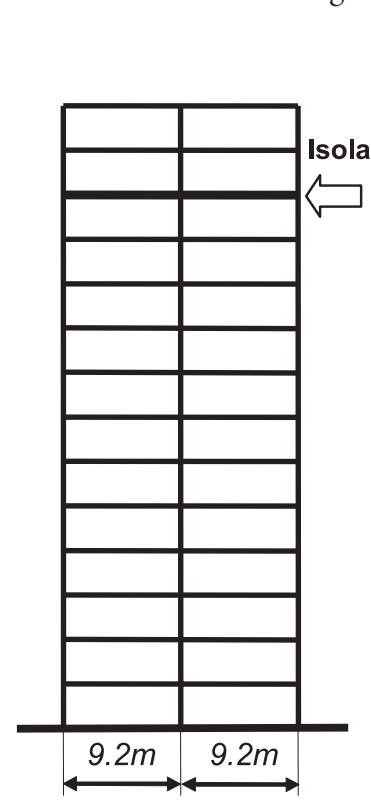

(a) ' $12+2$ ' Model

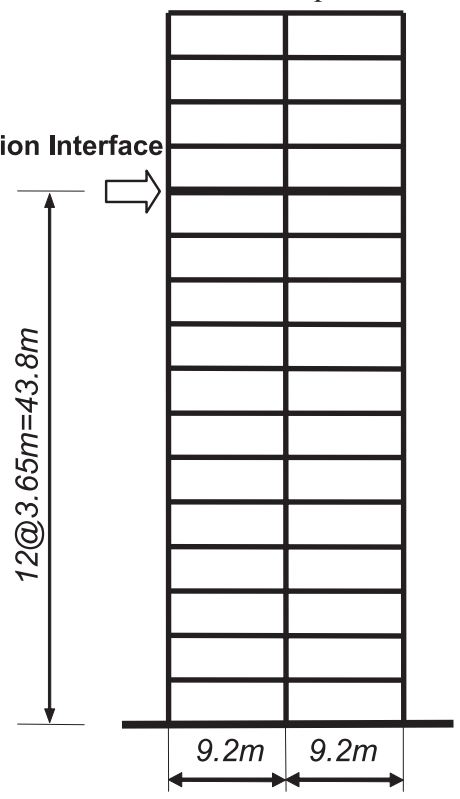

(b) ' $12+4$ ' Model
Figure 11. 12 Story+ Two-Bay Reinforced Concrete Frames

Table 4. Member Sizes of the Structure

\begin{tabular}{c|c|c}
\hline Members & Level & Dimensions $(\mathrm{mm})$ \\
\hline \multirow{4}{*}{ Beams } & $1-6$ & $900 \times 400$ \\
\cline { 2 - 3 } & $7-8$ & $850 \times 400$ \\
\cline { 2 - 3 } & $9-12$ & $800 \times 400$ \\
\hline \multirow{3}{*}{$\begin{array}{c}\text { Exterior } \\
\text { Columns }\end{array}$} & $1-6$ & $775 \times 500$ \\
\cline { 2 - 3 } & $7-8$ & $750 \times 500$ \\
\hline \multirow{3}{*}{$\begin{array}{c}\text { Interior } \\
\text { Column }\end{array}$} & $9-12$ & $650 \times 500$ \\
\cline { 2 - 3 } & $1-6$ & $800 \times 800$ \\
\cline { 2 - 3 } & $7-8$ & $725 \times 725$ \\
\hline
\end{tabular}

Table 5. Table 5. Dynamic properties of 12 -story existing structure

\begin{tabular}{c|c|c}
\hline Item & Main System & Unit \\
\hline Weight & 19,190 & $\mathrm{kN}$ \\
\hline $1^{\text {st }}$ Modal Mass & 1,514 & $\mathrm{kN}-\mathrm{s}^{2} / \mathrm{m}$ \\
\hline Natural period & 1.88 & $\mathrm{sec}$ \\
\hline Frequency & 0.531 & $\mathrm{~Hz}$ \\
\hline Damping Ratio & 0.05 & - \\
\hline $1^{\text {st }}$ Modal Amplitude & 1.36 & - \\
\hline
\end{tabular}

Upper two stories (' $12+2$ ' model) and four stories (' $12+4$ ' model) are isolated for the control of 12-story model and this means that $24 \%$ and $40 \%$ masses are added to the models respectively. Based on the dynamic properties of the existing 12-story frame (Table 5), the optimal parameters for TMD systems are listed in Table 6.

According to the results from the 2-DOF analysis, the SA80TMD was used under Kern County earthquake (low suite) and the SA40TMDs are used as SATMD control strategies under Imperial Valley (medium suite) and Kobe (high suite). The total value of $\mathrm{kM} 2$ opt is allocated to rubber bearing stiffness and the stiffness of the SA resettable device for the given SATMD building systems. The maximum SA actuator force is set at $400 \mathrm{kN}$, which represents $2.6 \%$ of the structural weight of $19,190 \mathrm{kN}$ for 12 -story structures. For the PTMD building system, the value of cM2opt is used as the damping coefficient of the viscous damper along with the value of kM2opt.

This application study is based on the design spectrum study (Chey et al., 2010), which assumes linearity traditionally used. Linear structure models give a good indication of the overall structure response without computational intensity of more realistic nonlinear studies. Hence, they provide good initial indications of the efficacy of any design approach. It is also important to note that such 12-story building structure used here (Jury, 1978) is designed to rarely yield at design level events per existing standards. Thus, the parametric optimization approach should yield good results for comparing overall performance with the analyses used.

Table 6. Table 6. Parameters for TMD building systems

\begin{tabular}{c|c|c|c|c|c}
\hline Model & $\mu$ & $\mathrm{f}_{\mathrm{M} 2 \mathrm{opt}}$ & $\xi_{\mathrm{M} 2 \mathrm{ppt}}$ & $\begin{array}{c}\mathrm{k}_{\mathrm{M} 2 \mathrm{opt}} \\
(\mathrm{kN} / \mathrm{m})\end{array}$ & $\begin{array}{c}\mathrm{C}_{\mathrm{M} 2 \mathrm{opt}} \\
(\mathrm{kN}-\mathrm{s} / \mathrm{m})\end{array}$ \\
\hline $\operatorname{PTMD}(12+2)$ & 0.31 & 0.684 & 0.716 & 2,448 & 1,535 \\
\hline $\operatorname{SATMD}(12+2)$ & 0.31 & 0.684 & - & 2,448 & - \\
\hline $\operatorname{PTMD}(12+4)$ & 0.52 & 0.568 & 0.842 & 2,814 & 2,498 \\
\hline $\operatorname{SATMD}(12+4)$ & 0.52 & 0.568 & - & 2,814 & - \\
\hline
\end{tabular}

\subsection{Performance results}

The analytical results for the described buildings are obtained to check the performance of each structural control case. To investigate the efficiency of the applied control systems, the response of No TMD, PTMD, and SATMD are compared in all floors and the response envelopes are presented in Figures 12 to 14. The peak relative displacements, total accelerations, interstory drift ratios and story shear forces for all floors are calculated as control effectiveness indices.

Overall, it is observed that the SATMD control provide satisfactory reductions and control performance is clearly dependent on the earthquakes. The SATMD building system is superior under Kern County (low suite) and Imperial Valley (medium suite), and the PTMD building system shows better reductions under Kobe (high suite). 
The maximum displacements of each level increase steadily over the height of the level. The envelopes of the interstory drifts are reasonably uniform and the drifts are decreased over the $12^{\text {th }}$ floor to $16^{\text {th }}$ floor, whereas the relatively large drifts can be found in upper part of middle levels. Overall, the SATMD systems present more reliable and constant interstory drift demands along the height of the existing 12-story structures. In the Kern County (low suite) and Imperial Valley (medium suite) earthquakes, for example, the SATMD systems show the drift demands close to $0.35 \%$ and $1 \%$ at most of the floors respectively.

The story shear forces show good reductions with the SATMD building systems based on the results from the maximum displacements. Especially, in spite of adding $24 \%$ mass $(12+2)$ and $40 \%$ mass $(12+4)$ to the buildings, the method of construction that uses TMD (PTMD and SATMD) at the interface actually reduces the seismic demand in the lower original stories by $25 \sim 55 \%$ under the all levels of the earthquake records considered.

Added viscous dampers of the PTMD systems have the benefit of being capable of reducing the acceleration demands on the structure while resulting in more even distribution over the height of the structures. Meanwhile, the acceleration responses of the isolated stories of the upper segment have a significant reduction in all TMD cases. The reason for these reductions is that the upper segment is isolated from the main structure, so base excitation is not directly transmitted to the separated upper portion of the TMD.
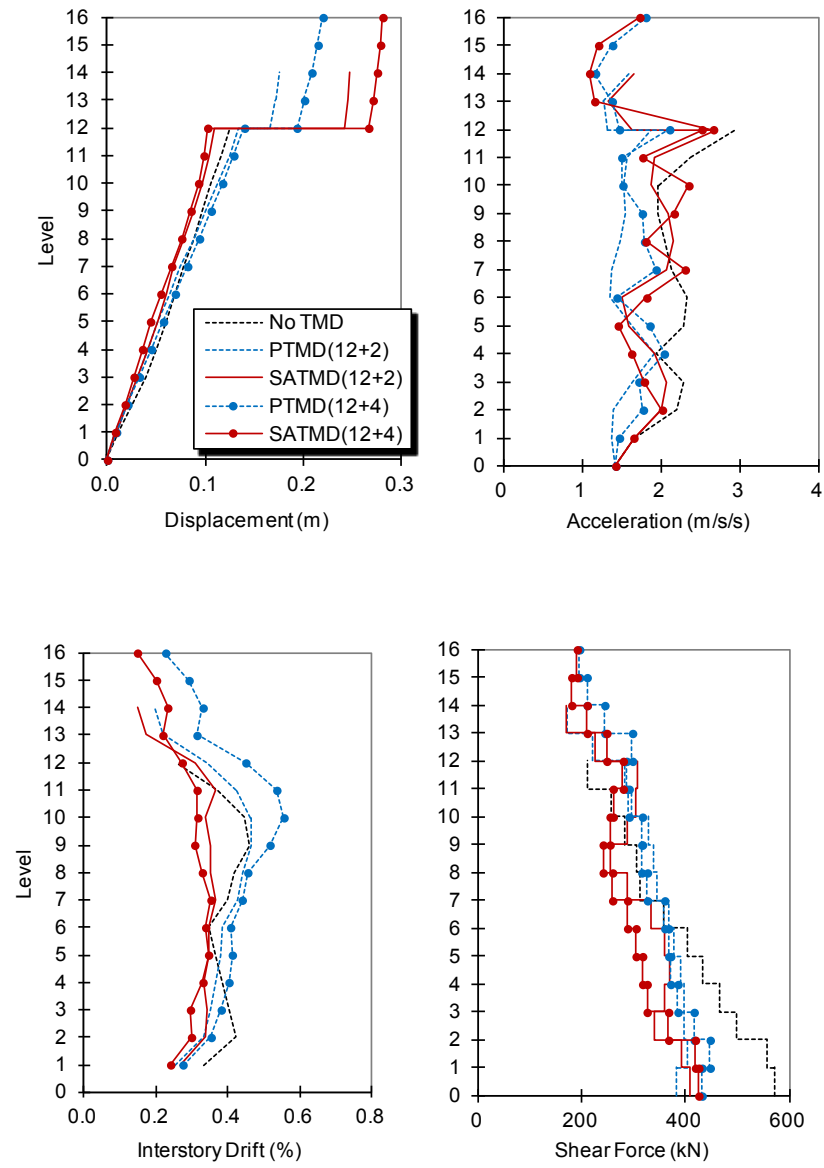

Figure 12. Control performance of ' $12+2$ ' and ' $12+4$ ' models (Kern County 1952 / Low Suite)
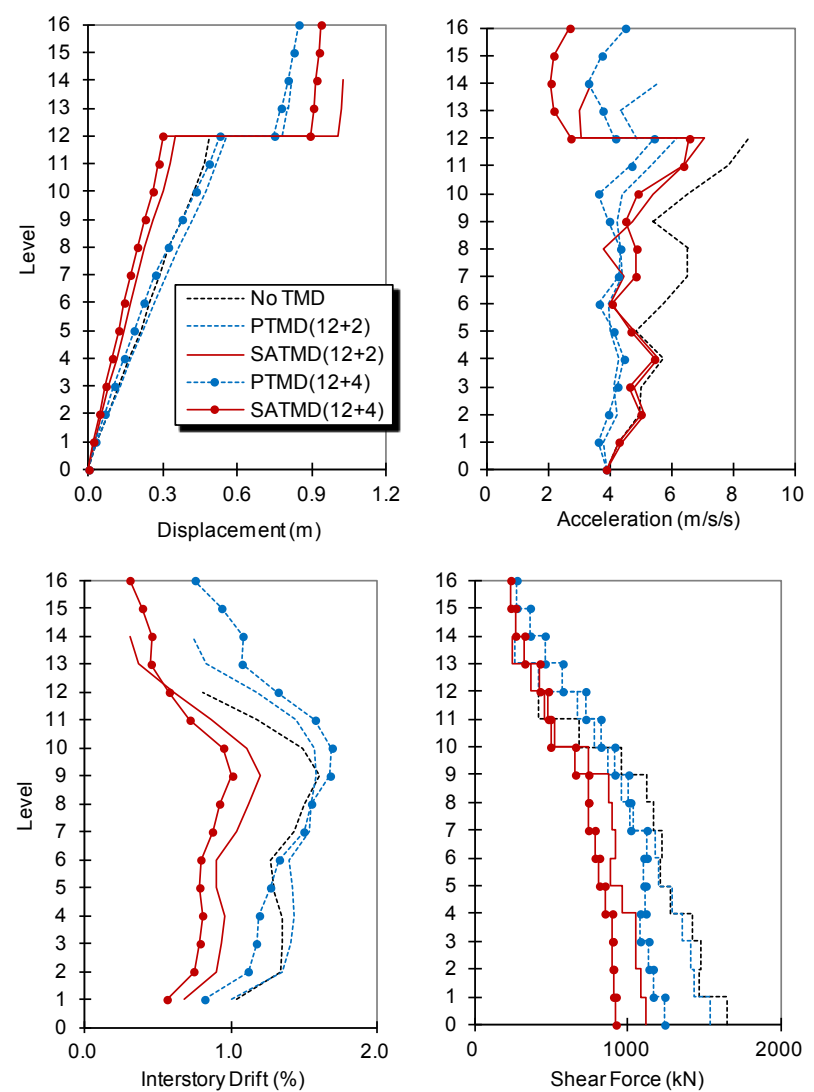

Figure 13. Control performance of ' $12+2$ ' and ' $12+4$ ' models (Imperial Valley 1979 / Medium Suite)
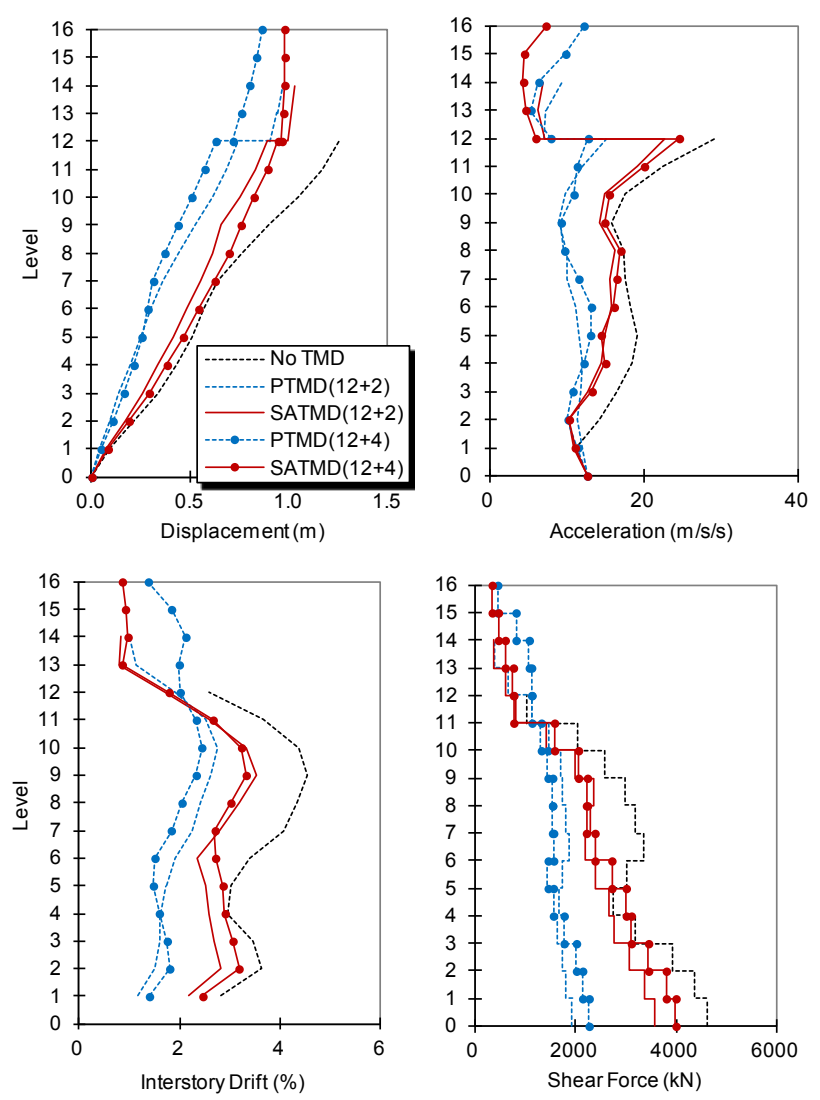

Figure 14. Control performance of ' $12+2$ ' and ' $12+4$ ' models (Kobe 1995 / High Suite) 
In all cases, it should be noted again that PTMD results represent optimal exact tuning. Such exact tuning may not be possible, practically, due to construction or load variability, as well as degradation over time. From the parametric results of this study, the design 'range' of effective SATMD system was derived and then adopted as a practical control scheme. Semi-active solutions are also not constrained by the optimal tuning stiffness for the TMD like the passive case (Chey et al., 2010). Thus, the design approach of SATMD system is different from the PTMD system and the SATMD system is relatively easier to design as no tuning is required. Hence, the SATMD results would appear much better given their broad control band and adoptability.

The efficacy of the suggested SATMD system is OK for small to medium sized events. However, it is beyond the scope of this study to analyze non-linearity in very large event here (Kobe) but is addressed, showing small impacts. In particular, to reduce optimization variables and optimization non-convexity and nonlinearity, we have initially hypothesized that the optimal PTMD stiffness represents a good or acceptable design baseline. This choice is much like choosing a starting value for any non-linear optimization problem. In this case, we have chosen start value and a parametric approach to SATMD stiffness contributions to define the variable space and yield a highly computationally efficient approach to designing such systems.

\section{CONCLUSIONS}

This paper presents preliminary study on the seismic response of a multi-story semi-active tuned mass damper building system to various intensity level seismic earthquake records. A 2-DOF undamped model has been used to explore the efficacy of parametric control and control abilities of the TMD (PTMD and SATMD) building systems. A 12-story, two-bay reinforced concrete framed structure has been developed in Ruaumoko to demonstrate the effects of the TMD building systems.

From the 2-DOF model analyses, the validity of the optimal parameters was demonstrated and a reasonable stiffness allocation between the resettable device and rubber bearings was investigated. More importantly, the 2-DOF analyses show the efficacy of this methodology to create reliable structural control management.

Based on the 2-DOF analyses, the 12-story case study showed that the practical adoptability of the TMD building systems. From the performance results based on the several response indices, the SATMD building systems present better control performance to low and medium suite records, whereas the PTMD building systems present better control performance for high suite record and due to the contribution of the viscous damping. However, it should be noted that the PTMD results are optimal, but not necessarily practical. Specifically, the 50-70\% damping ratio might not be really achieved. Thus, similar SATMD results indicate that optimal level solutions can be obtained without resorting to unrealistically large viscous damper values.

From the results of additional ' $12+2$ ' and ' $12+4$ ' story retrofit case studies, SATMD systems show significant promise for application of structural control where extra stories might be added. They offer unique advantages over PTMD systems in obtaining consistent response reductions over broad ranges and types of ground motions at realistic seismically important structural natural frequencies. They are thus more robust to ground motion variation, as they provide tighter ranges across each suite.

This research has demonstrated the validity of a SATMD segmental building system for consideration in future design and construction. Further studies are underway to investigate the seismic response of the structures with various periods under a number of acceleration records of actual earthquakes.

Even though this study does not provide exact design criteria, the aim of this analysis is to quantify the fundamental qualitative benefit of these TMD systems by examining both the efficacy of the modified structural configuration and the use of resettable devices in that approach. The response features obtained in this linear analysis can be used as the initial design reference for the further studies investigating inelastic seismic response for more realistic nonlinear structures.

\section{REFERENCES}

Barroso, L.R., Chase, J.G., and Hunt, S. (2003) "Resettable smart dampers for multi-level seismic hazard mitigation of steel moment frames." Journal of Structural Control, 10(1): 41-58.

Bobrow, J.E., and Jabbari, F. (1997) "A High-performance Semiactive Controller for Structural Vibration Suppression." Smart Structures and Materials, SPIE, 3041.

Carr, A.J. (2007) Ruaumoko - Computer Program Library, Department of Civil Engineering, University of Canterbury, Christchurch, New Zealand.

Charng, P.H. (1998) "Base isolation for multistory building structures." PhD Thesis, Department of Civil Engineering, University of Canterbury, Christchurch, New Zealand.

Chase, J.G., Barroso, L.R., and Hunt, S. (2004) "The impact of total acceleration control for semi-active earthquake hazard mitigation." Engineering Structures, 26(2): 201-209.

Chase, J. G., Mulligan, K. J., Gue, A., Alnot, T., Rodgers, G., Mander, J. B., Elliott, R., Deam, B., Cleeve, L., and Heaton, D. (2006) "Reshaping hysteretic behaviour using semi-active resettable device dampers." Engineering Structures, 28(10), 1418-1429.

Chey, M.H., Chase, J.G., Mander, J.B. and Carr, A.J. (2010) "SemiActive Tuned Mass Damper Building Systems: Design." Earthquake Engineering \& Structural Dynamics, 39(2): 119-139.

Hunt, S.J. (2002) "Semi-active smart-dampers and resettable actuators for multi-level seismic hazard mitigation of steel moment resisting frames." ME Thesis, Department of Mechanical Engineering, University of Canterbury, Christchurch, New Zealand.

Jabbari, F., and Bobrow, J. E. (2002) "Vibration suppression with resettable device." Journal of Engineering Mechanics, 128(9): 916-924.

Jury, R.D. (1978) "Seismic load demands on columns of reinforced concrete multistorey frames." ME Thesis, Department of Civil Engineering, University of Canterbury, Christchurch, New Zealand.

Mulligan, K., Chase, J., Gue, A., Alnot, T., Rodgers, G., Mander, J., Elliott, R., Deam, B., Cleeve, L., and Heaton, D. (2005) "Large Scale Resetable Devices for Multi-Level Seismic Hazard Mitigation of Structures. Proc. 9th International Conference on 
Structural Safety and Reliability (ICOSSAR), Rome, Italy, 19-22.

NZS4203 (1976) "New Zealand Standard; Code of Practice for General Structural Design and Design Loadings for Buildings." Standards Association of New Zealand (SANZ).

NZS4203 (1992) "New Zealand Standard; Code of Practice for General Structural Design and Design Loadings for Buildings." Standards Association of New Zealand (SANZ).

Pan, T.-C., Ling, S.-F., and Cui, W. (1995) "Seismic response of segmental buildings." Earthquake Engineering \& Structural Dynamics, 24(7): 1039-1048.

Pan, T.C., and Cui, W. (1998) "Response of segmental buildings to random seismic motions." ISET Journal of Engineering Technology, 35(4): 105-112.

Paulay T. (1976) "Moment redistribution in continuous beams of earthquake resistant multistory reinforced concrete frames." Bulletin of the New Zealand National Society of Earthquake Engineering, 9(4): 205-212.

Rodgers, G.W., Mander, J.B., Chase, J.G., Mulligan, K.J., Deam, B.L., and Carr, A.J. (2007) "Re-shaping hysteretic behaviour - Spectral analysis and design equations for semi-active structures." Earthquake Engineering \& Structural Dynamics, 36(1), 77-100.

Sadek, F., Mohraz, B., Taylor, A.W., and Chung, R.M. (1997) "A method of estimating the parameters of tuned mass dampers for seismic applications." Earthquake Engineering \& Structural Dynamics, 26(6): 617-635.

Sommerville, P., Smith, N., Punyamurthula, S., and Sun, J. (1997) "Development of ground motion time histories for Phase II of the FEMA/SAC steel project." SAC Background Document Report No. SAC/BD-97/04

Tian, Z., Qian, J., and Zhang, L. (2008) "Slide roof systems for dynamic response reduction." Earthquake Engineering \& Structural Dynamics, 37(4): 647-658.

Villaverde, R. (2002) "Aseismic roof isolation system: Feasibility study with 13-story building." Journal of Structural Engineering, 128(2): 188-196.

Warburton G.B. (1982) "Optimum absorber parameters for various combinations of response and excitation parameters." Earthquake Engineering \& Structural Dynamics, 10: 381-401.

(Received January 31, 2012/Accepted August 1, 2012) 